\title{
The linear dependence of GHz scintillation on electron density observed in the equatorial anomaly
}

\author{
J. A. Whalen \\ 200 Wood Street, P.O. Box 97, Woodville MA 01784, USA
}

Received: 16 September 2008 - Revised: 11 February 2009 - Accepted: 27 March 2009 - Published: 9 April 2009

\begin{abstract}
The dependence of scintillation caused by equatorial bubbles on the electron density in the post sunset anomaly cannot generally be determined because the irregularities that produce the scintillation obscure the measurement of the electron density. This work addresses this problem with the introduction of a new technique based on the finding that anomaly electron density is so regular that its LT distribution can be fit by a polynomial over its post-sunset extent. By means of this polynomial, gaps in the data caused by the irregularities can be inferred where otherwise not measurable. Observations are of amplitude scintillation at $1.5 \mathrm{GHz}$ from the Marisat satellite and of $\mathrm{Nm} \mathrm{F} 2$ measured by ionospheric soundings at Ascension I. in Mar 2001 during solar maximum. The result is that the well defined maximum of the S4 scintillation index is a linear function of coinciding $N m \mathrm{~F} 2$. Evidence that supports this result has been found in 3 differing cases: The first finds that both $\mathrm{S} 4$ and $N m \mathrm{~F} 2$ decrease linearly with LT; the second, that both occurrence of scintillation and $N m \mathrm{~F} 2$ increase linearly with $\mathrm{F} 10.7 \mathrm{~cm}$ solar flux; the third finds a linear relation between occurrence of scintillation at $137 \mathrm{MHz}$ and $\mathrm{NmF} 2$. The importance of these results is that scintillation is caused by the bubble, but its magnitude is determined by the magnitude of the electron density that the bubble intersects. A further implication is that, given the unpredictability of bubbles, if $N m \mathrm{~F} 2$ could be predicted, the maximum level of S4 possible could be predicted if a bubble were to occur.
\end{abstract}

Keywords. Ionosphere (Equatorial ionosphere; Ionospheric disturbances; Ionospheric irregularities)

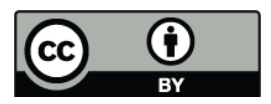

Correspondence to: J. A. Whalen (jwhalen11@verizon.net)

\section{Introduction}

The study of RF scintillation, which is caused by irregularities that scatter RF transmissions passing through the ionosphere, has a long history starting with observations of emissions by radio stars (e.g. Aarons, 1997). The study gained particular importance with the launch of satellites because of the disruption of transionospheric RF communication, radar and global positioning. Particularly disruptive is the scintillation in the post sunset equatorial ionosphere caused by irregularities accompanying equatorial spread $\mathrm{F}$, principally bottomside spread $\mathrm{F}$ near the dip equator and the equatorial plume or bubble that rises in altitude so as to extend from equator to the post-sunset equatorial anomaly (Kudeki and Bhattacharyya, 1999; Whalen, 2000). Because of these disruptive effects, much of the study of the equatorial aeronomy is justified by the need to understand and predict this scintillation. The irregularities are perturbations of the ionosphere, but are often so extreme that they prevent simultaneous measurement of the electron density. Even so, past work indicates that scintillation is dependent on the ionosphere, so that its properties cannot be understood independent of the properties of the medium which the irregularities perturb.

This paper is the first attempt to quantify the dependence of scintillation on electron density. Its impetus is the evidence of such dependence found by Aarons et al. (1981) that scintillation was greater in the anomaly than at the equator, which they attributed to the electron density being greater in the anomaly than at the equator. The cause of scintillation was identified as equatorial bubbles in both equatorial and anomaly regions by a set of coincident airborne ionospheric soundings and optical images by Weber et al. (1978, 1982). Observation of individual bubbles by the SCINDA program (Basu and Groves, 2001) found scintillation to be greater at the anomaly than at the equator by simultaneous measurements in both locations, further supporting the difference to be due to the difference in electron density. 


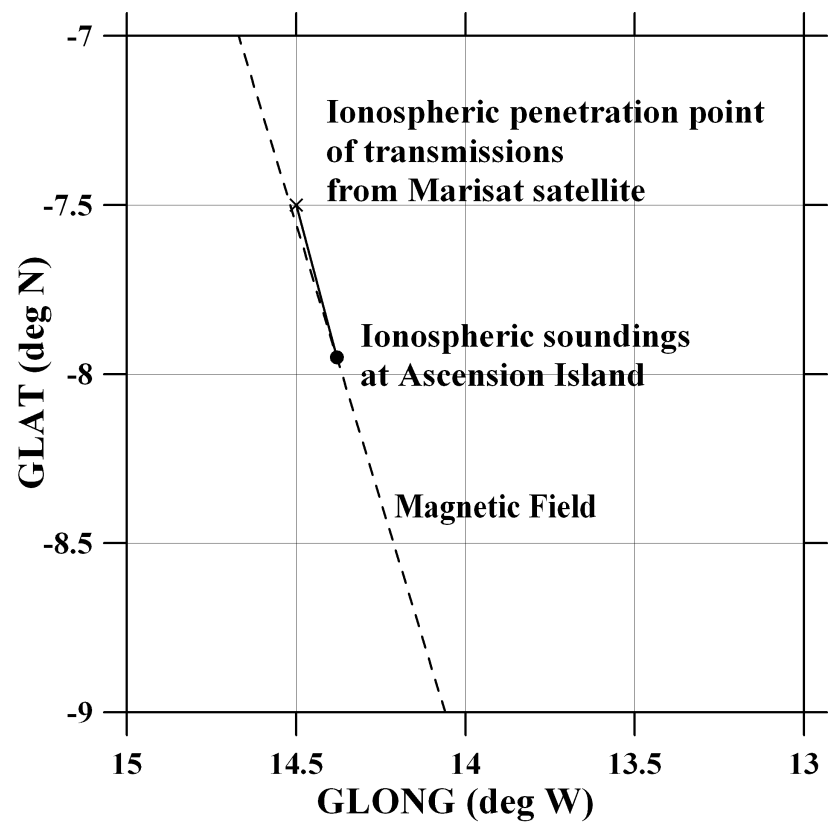

Fig. 1. Location of the measurements. Transmissions from the Marisat satellite are received along the ray path (solid line), aligned approximately along the magnetic field (dashed line) with the effective ionospheric penetration point is shown as the X. Ionospheric soundings are made at the zenith at Ascension I. shown as the solid circle.

Scintillation at GHz was found to be associated with the boundaries of the bubble using joint measurements by the RPA analyzer on the AE-E satellite together with the Jicamarca ISR by Basu et al. (1980) and ground based measurements by Basu et al. (1983). The latter paper found the more intense $\mathrm{GHz}$ scintillations to be associated with bubbles having large depletions that were immersed in a background of high ambient electron density, a result that is further evidence of the dependence of scintillation on electron density.

The dependence described above is not quantitative because measurement of electron density particularly by ionospheric soundings is generally blocked by the irregularities that cause the scintillation. In order to overcome this difficulty this paper reports a new approach to the measurement of $N m \mathrm{~F} 2$ under these conditions. It is based on the result of these observations that the post sunset anomaly $\mathrm{F}$ layer has on each day a LT dependence of $N m \mathrm{~F} 2$ is so regular that it can be fit by a single polynomial. Because this function can bridge the gaps in the data caused by the irregularities from the bubble, electron density can be inferred where the irregularities prevent its direct measurement.

The result is a linear relation between the well-defined scintillation maxima and the coinciding $N m$ F2. The search for additional evidence in support of this dependence has found two cases where both electron density and $\mathrm{GHz}$ scintillation are determined separately as functions of other param- eters, LT on the one hand and solar flux on the other. A third case indicates similar support but for scintillation at VHF.

In outline, Sect. 2 describes experimental details. Section 3 , a case study of the interrelation of the measurements. Section 4, the approach to measurement. Section 5, the results and discussion. Section 6, a summary.

\section{Experiment}

Observations were made during an expedition by the Air Force Research Laboratory to the Ascension Island station of the SCINDA Network (Basu and Groves, 2001) on 13-31 March 2001. Conditions were solar maximum with F10.7 cm varying between 135 and 275 solar flux units. Magnetic activity varied from $\operatorname{sum} K_{p}$ of 4 to 61 .

Scintillation on the $1.5 \mathrm{GHz}$ transmissions from the Marisat satellite was measured at 5-min intervals by the S4 index, the standard deviation divided by the average signal intensity (Briggs and Parkin, 1963). Ionospheric soundings were by a Digisonde (Reinisch et al., 1989) making ionograms $1-30 \mathrm{MHz}$ also at $5 \mathrm{~min}$ intervals. Ionograms were made available by the University of Massachusetts at Lowell and were scaled manually. The body of data was 180 ionograms for each of the 14 days scaling at least 2 parameters, $f o \mathrm{~F} 2$ and $h^{\prime} F$ comprised more that 5000 individual measurements. The resolution and continuity of the measurements has provided the basis for the approach to the solution here.

The location of the measurements is shown in Fig. 1. At Ascension the ionospheric soundings are located near the ionospheric penetration point of the satellite transmissions. There were 11 days during which both $\mathrm{S} 4$ associated with bubbles and $N m \mathrm{~F} 2$ where measured. 3 days were magnetically disturbed and had no bubbles but $N m \mathrm{~F} 2$ was measurable. On 5 days there were insufficient measurements of $N m \mathrm{~F} 2$.

\section{Case study illustrating the measurements}

The ionospheric and scintillation measurements made on 1617 March 2001 are shown together as a function of LT in Fig. 2. Panel (a) records the sounder measurements of $h^{\prime} F$, the virtual height of the F-layer. The radar range echoes from 4 bubble boundaries are also shown in this panel.

Panel (b) records $N m \mathrm{~F} 2$, the maximum electron density of the F-layer derived from the measurements of $f o \mathrm{~F} 2$, the maximum plasma frequency. The solid curve is the polynomial fitting the data from 18:30 to 01:30 LT.

Panel (c) records the scintillation index S4 measured on the $1.5 \mathrm{GHz}$ transmissions from the Marisat satellite. Each of the 4 bubble boundaries produces a peak in $\mathrm{S} 4$ as it passes over Ascension. 

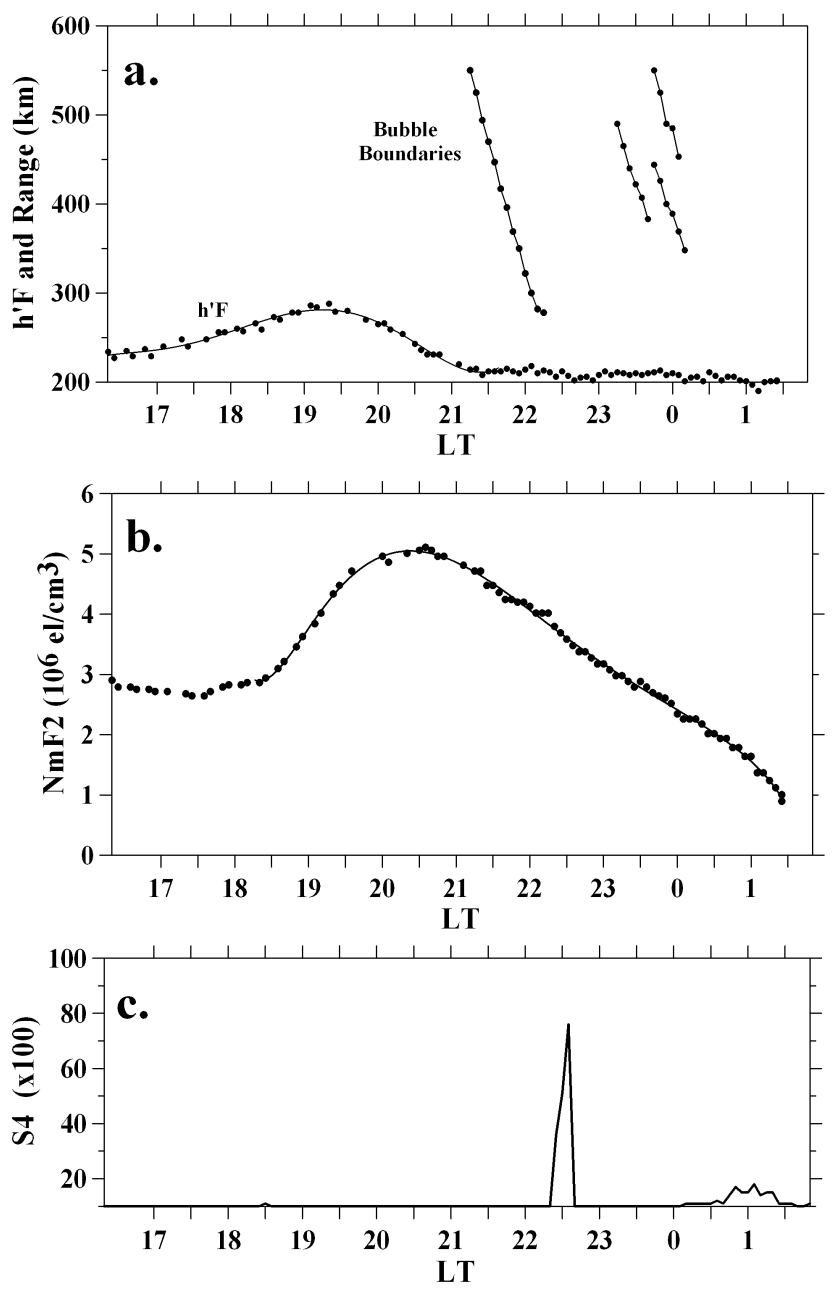

Fig. 2. The ionospheric and scintillation observations on 1617 March 2001. (a) $h^{\prime} F$ and Range, F-layer virtual height and radar range to bubble boundaries measured by the sounder. (b) $N m \mathrm{~F} 2$, maximum $\mathrm{F}$ layer electron density also measured by the sounder. Solid curve is the polynomial fit to the data. (c) S4, Scintillation index at $1.5 \mathrm{GHz}$.

\section{Approach to measurement}

The case study described above is not typical because $N m \mathrm{~F} 2$ was measurable when the bubble was overhead, whereas it usually disturbs the ionosphere so much that its electron density cannot be measured simultaneously with the scintillation. The approach here is the outcome of the detailed measurements of many cases over $10 \mathrm{~h}$ durations at $5 \mathrm{~min}$ intervals: the LT distribution of $N m \mathrm{~F} 2$ on each day is so regular that it can be fit over most of its post-sunset extent by a polynomial in LT unique to that day. Figure $2 b$ is an example of such a fit.

The significance of this regularity appears on 14-15 March 2001 in which $N m F 2$ is not measurable in the interval from $21: 55$ to $23: 25 \mathrm{LT}$ as the result of the disturbance caused by

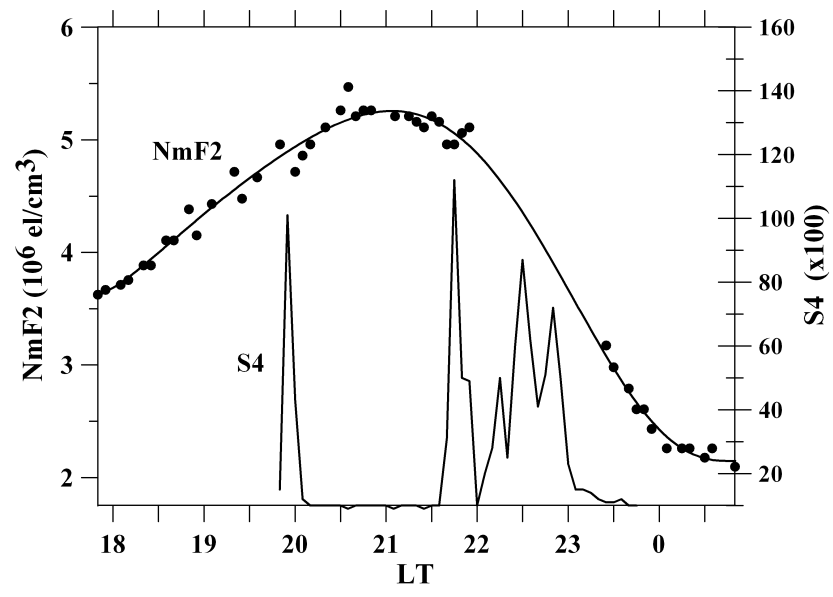

Fig. 3. Ionospheric and scintillation observations on 14-15 March 2001 showing the interpolation of $N m \mathrm{~F} 2$ where the scintillation irregularities obscure its direct measurement.

bubbles, the presence of which is indicated by the occurrence of S4 (Fig. 3). The polynomial shown by the solid curve that fits the data from 17:55 to 00:45 LT will be used to interpolate $N m \mathrm{~F} 2$ in the interval so as to yield values simultaneous with S4.

This approach is consistent with the results of Basu et al. (1983) who found bubbles in situ to be depletions an the otherwise smoothly varying ambient plasma in which strong scintillation occurred at bubble boundaries which were sharp negative gradients in electron density. In the following each well resolved peak in S4, S4max, will be measured, and the $N m \mathrm{~F} 2$ that coincides with it, if not measurable, will be inferred by polynomial fit illustrated here.

\section{Results and discussion}

\subsection{This approach}

The 47 determinations of S4max and coinciding $N m \mathrm{~F} 2$ are plotted in Fig. 4. The straight line fit, has equation,

$\mathrm{S} 4 \mathrm{max}=25.6 \times N m \mathrm{~F} 2-20.4$

where $\mathrm{S} 4$ is $\mathrm{x} 100$ and $\mathrm{NmF} 2$ is in $10^{6} \mathrm{el} / \mathrm{cm}^{3}$. The quality of the fit is $r^{2} \sim 0.8, s \sim 17$.

This result is evidence that scintillation is caused by the bubble, but that its magnitude is determined by the magnitude of the electron density that the bubble intersects.

Because this is an original result, it has the limitations of being an otherwise untested technique, and is based on 11 days observations all at solar maximum. Furthermore, the uncertainties are greatest where the magnitudes are greatest and therefore most important. However if scintillation has this dependence on electron density, it can be expected to appear in other investigations. To that end other observations 


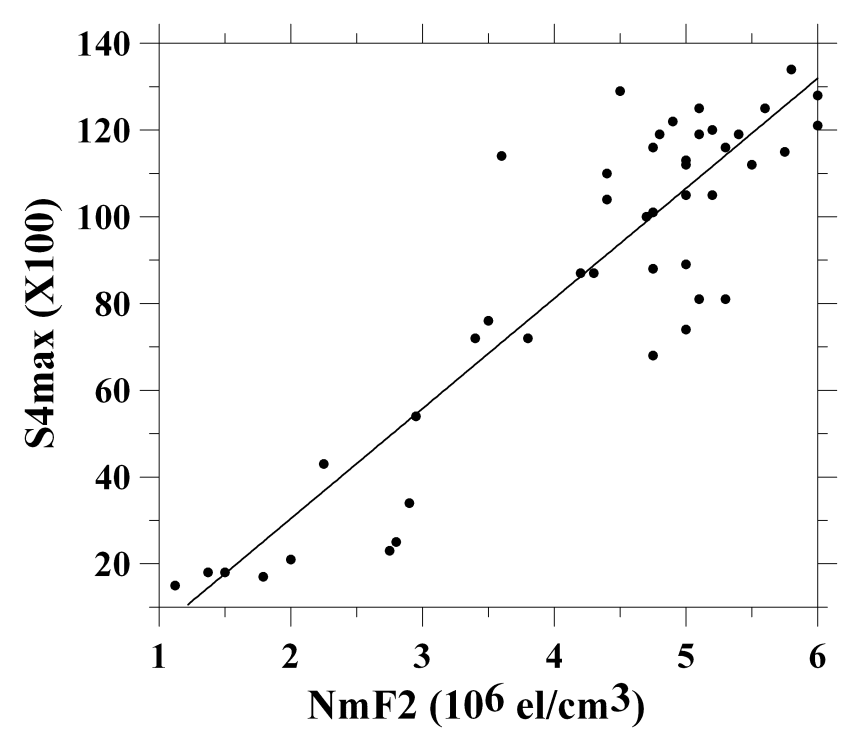

Fig. 4. Variation of $\mathrm{S} 4 \mathrm{max}$ with coincident measured/inferred NmF2 for 13-31 March 2001.

have been examined to see if they are consistent with this result.

\subsection{Other evidence}

The dependence of $\mathrm{S} 4$ on $N m \mathrm{~F} 2$ is found in three additional cases. The first additional case considers the same data set described above, but analyzes $N m \mathrm{~F} 2$ and $\mathrm{S} 4 \mathrm{max}$ as functions of the LT parameter. The second re-analyzes past independent measurements in which $N m \mathrm{~F} 2$ and $\mathrm{S} 4$ are determined as functions solar flux. The third measures occurrence of scintillation at VHF with $N m \mathrm{~F} 2$.

\subsubsection{LT dependence}

The measurements of Fig. 4 are re-examined here but as separate functions of LT. $N m \mathrm{~F} 2$ that coincides with $\mathrm{S} 4 \mathrm{max}$ is plotted vs. LT in Fig. 5a. The straight line fit shows it to decrease linearly with LT, evidently as the result of F layer recombination. In comparison, S4max vs. LT shown in Fig. 5b, also decreases linearly with LT, necessarily so because it has been found to be a linear function of $N m \mathrm{~F} 2$ as shown in Fig. 4.

If S4max vs. LT (Fig. 5b) were viewed by itself, the interpretation might be that $\mathrm{S} 4$ decreases because the irregularities decay with time. However, when seen together with the decrease of coinciding $N m \mathrm{~F} 2$ with LT, it is evidence that $\mathrm{S} 4$ decreases with LT because the ionospheric medium in which the irregularities form decreases with LT.

In apparent contrast to these results, Hysell and Kelley (1997) found scintillation to decrease with LT as the result of irregularity decay. The choice between the two effects, or whether there is a combination of the two, cannot be explicitly determined in the present observations. However,
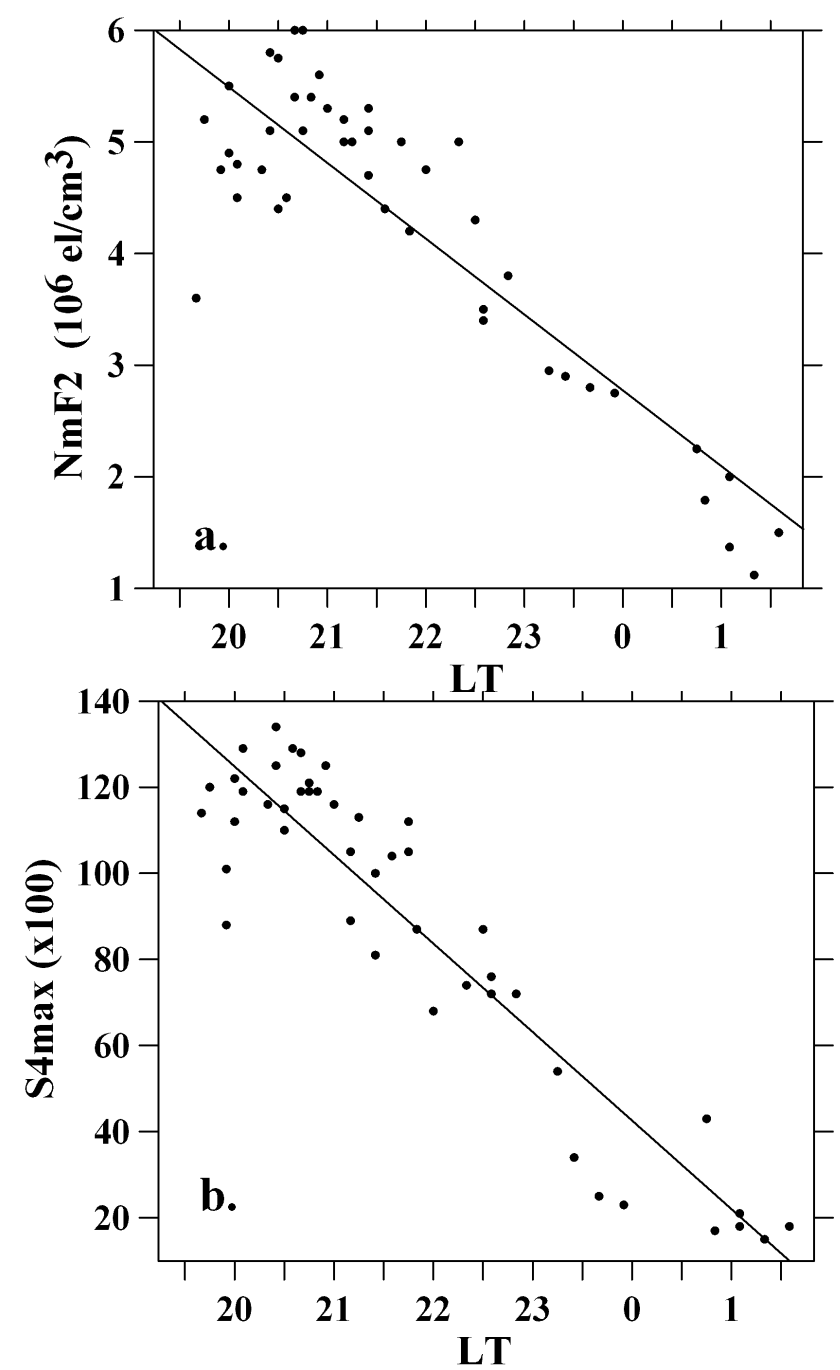

Fig. 5. $N m \mathrm{~F} 2$ and $\mathrm{S} 4 \mathrm{max}$ data as functions of LT.

other work has found that differences in scintillation can result from differences in $N m \mathrm{~F} 2$ alone. In particular, Aarons et al. (1981) found that scintillation was greater at the anomaly than at the equator and attributed it to the fact that the electron density was greater at the anomaly than at the equator. Furthermore, SCINDA (Basu and Groves, 2001) also found lower S4 at the equator than at the anomaly but for individual bubbles. Because the SCINDA observations were simultaneous in equator and anomaly, the regularities were of the same age so that differences in decay could not account for the difference in the magnitude of the scintillation.

\subsubsection{Solar flux dependence}

A second example in the effort to find corroboration of the dependence of $\mathrm{S} 4$ on $N m \mathrm{~F} 2$ uses the relation of both to solar flux. In addition it does not depend on the technique of interpolating $N m \mathrm{~F} 2$ nor is it limited to solar maximum. 
$\mathrm{NmF2}$

$\mathrm{Nm} \mathrm{F} 2$ at the anomaly maximum is linearly dependent on F10.7 cm solar flux, as measured by individual month at locations worldwide during the 13 years starting at solar maximum at 1957, through solar minimum to the following solar maximum at 1969 (Whalen, 2004). Each of the 12 months showed this linearity, but with a slope that is greatest in the equinoxes, least near June solstice, and intermediate near December solstice. The same result was found at each location, but the magnitude of the slope differed principally by latitude. Although $N m \mathrm{~F} 2$ was not measured at Ascension Island during the period of the S4 measurements, based on all the other measurements it can be assumed also to have a linear dependence on solar flux that varied similarly by month.

The measurements of $N m \mathrm{~F} 2$ from Taipei and the $\mathrm{S} 4$ measurements from Ascension Island, as described below, are shown for the Sep-Dec period in Fig. 6.

\section{S4}

Evidence that S4 occurrence is linearly dependent on solar flux has been found in the measurements of the same $1.5 \mathrm{GHz}$ scintillation from Ascension I from maximum to minimum of solar cycle \# 21, from 1980 through 1985 as a result of a re-analysis of the data extracted from Fig. 3 of Basu et al. (1988).

In order to compare scintillation to solar flux, the approach is the same as that for electron density, namely to describe the dependence by month of scintillation on solar flux expressed as the percent occurrence of fade level $\geq 5 \mathrm{~dB}$ which is approximately the percent occurrence of $S 4 \geq 0.4$. The first panel in Fig. 6b shows the measurements as functions of solar flux on successive September months from 1980 (solar maximum) up to 1980 (solar minimum). The other panels show the same for October through December, months which have enough data to be analyzed. The relation is linear for each month, and the slope decreases in monthly order as it does for Nemax.

Although the results are not quantitative, they have following interpretation: $N m \mathrm{~F} 2$ is a linear function of solar flux, and that S4 is also a linear function of solar flux is consistent with its being a linear function of $N m \mathrm{~F} 2$, which is the principal finding of this work.

\subsection{VHF scintillation}

The third example revisits the results of Huang (1970) which show evidence that supports the current findings in a very different frequency regime, technique and location. Huang measured occurrence of scintillation in the anomaly at $137 \mathrm{MHz}$ from transmissions from the Intelsat II-F2 satellite at Taipei $\left(25.0^{\circ} \mathrm{N}, 121.5^{\circ} \mathrm{E}\right)$ in relation to RSF measured with ionospheric soundings. He found good correlation between the two, but did not report $f o \mathrm{~F} 2$. Fortuitously this data

\section{a. $\mathrm{NmF2}$}

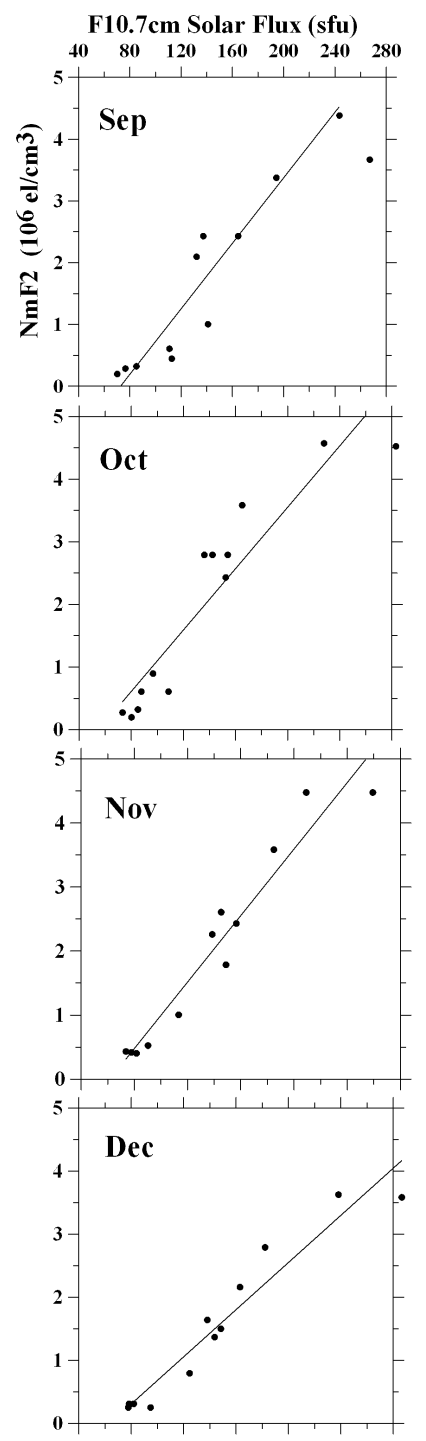

b. Scintillation

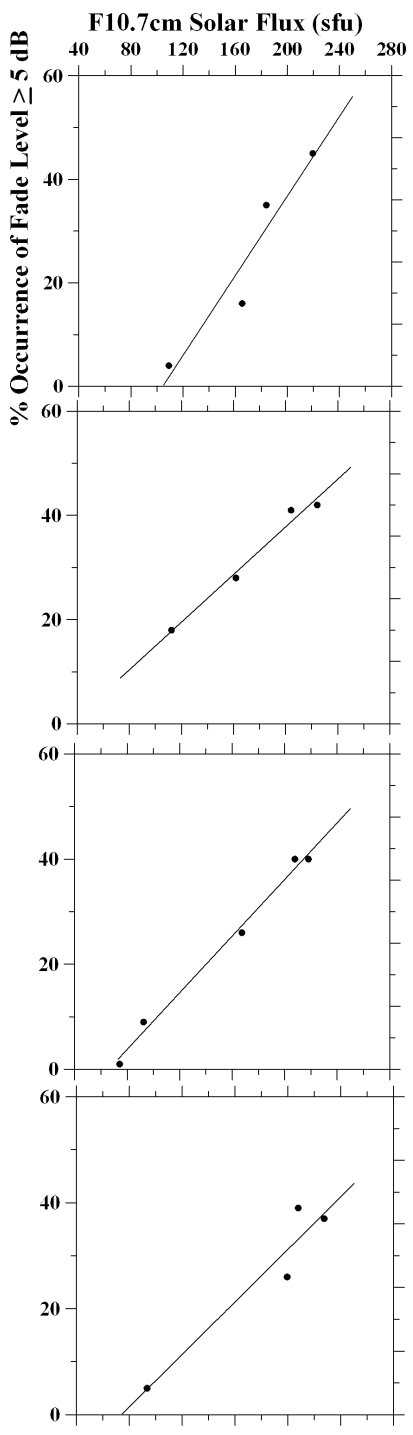

Fig. 6. $\mathrm{S} 4$ and $N m \mathrm{~F} 2$ dependence on solar flux from 1980 (solar maximum) through 1985 (solar minimum) from September to December. (a) $N m$ F2 from Taipei (Whalen, 2004). (b) $1.5 \mathrm{GHz}$ scintillation recorded at Ascension Island. \% occurrence of $>5 \mathrm{~dB}$ is approximately $\%$ occurrence of $\mathrm{S} 4>0.4$.

is available during the same period in the Taipei soundings of the monthly median $f o \mathrm{~F} 2$ at hourly intervals recorded in individual graphs and tabulations in the ionospheric data published by the National Oceanic and Atmospheric Administration as described in Whalen (2004).

Results from March-April 1969 in Fig. 7 show the percent occurrence of $137 \mathrm{MHz}$ scintillation to increase as a linear function of $N m \mathrm{~F} 2$. This result is consistent at $137 \mathrm{MHz}$ with that found at $1.5 \mathrm{GHz}$ shown in Fig. 4. In addition, the points labeled with hourly values of LT, show that both decrease jointly from 21:00 to 05:00 LT, evidencing a dependence on LT that is consistent with that shown in Fig. 5. 


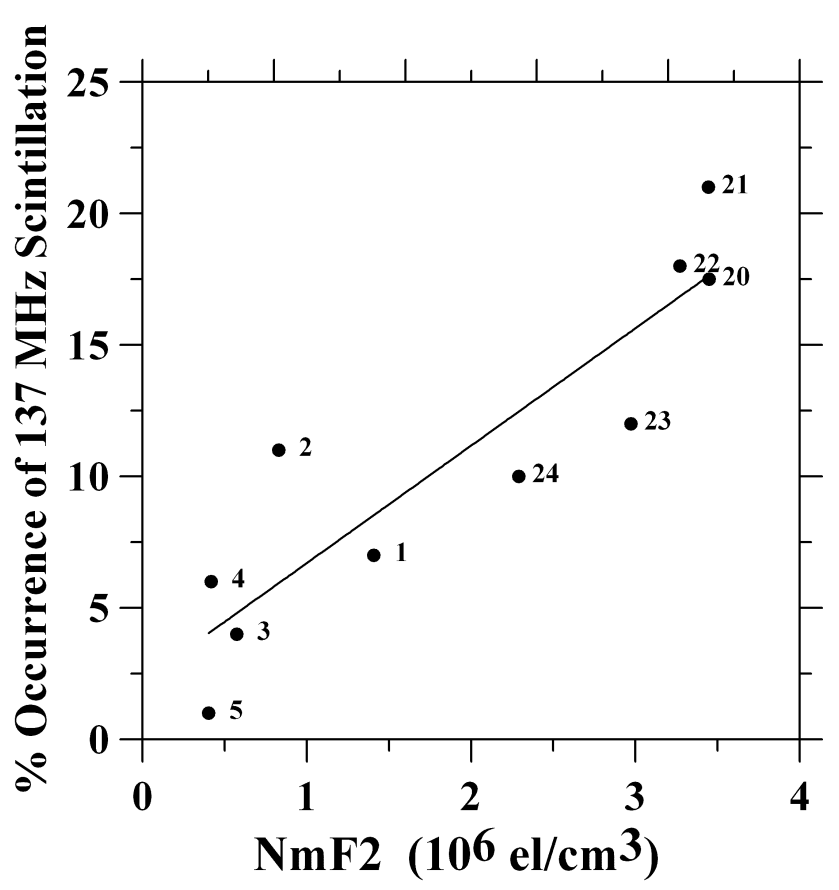

Fig. 7. Scintillation occurrence at $137 \mathrm{MHz}$ as a function of $\mathrm{NmF} 2$ as observed on March-April 1969 at Taipei. Labels on the points are the local times of the measurements.

\section{Summary}

The difficulty of determining electron density when its measurement is obscured by irregularities that cause scintillation has been addressed by a new technique. This technique is based on the finding that the anomaly F-layer electron density is normally so regular that a single polynomial can fit most of its post-sunset extent. Because this polynomial can interpolate gaps in the data caused by an equatorial bubble, $N m \mathrm{~F} 2$ that coincides with the scintillation can be inferred even when obscured by the bubble irregularities.

The measurements were done in the anomaly region at the Ascension Island station during solar maximum on March 2001. In this location ionospheric soundings are located near the ionospheric penetration point of the $1.5 \mathrm{GHz}$ transmissions from the Marisat satellite.

By means of this interpolation, $N m \mathrm{~F} 2$ that coincides with S4max can be inferred if not measurable directly. The result of 47 such coincidences is that $\mathrm{S} 4 \mathrm{max}$ is a linear function of the coinciding $N m \mathrm{~F} 2$.

The result is limited because it depends on an otherwise untested technique, a small number of observations and a single location, season and solar flux.

Therefore a search undertaken for other evidence under a variety of conditions has found it in 3 sets of observations that supports the existence of such a dependence. The first uses the same data set, but where $\mathrm{S} 4 \mathrm{max}$ and $N m \mathrm{~F} 2$ are recorded separately as functions of LT. Here $N m \mathrm{~F} 2$ decreases linearly with LT, evidently because recombination erodes the F layer progressively with LT. S4max also decreases linearly with LT, but indicates that it does so because the ionospheric medium in which the irregularities form decreases with LT.

The second is a separate study at the same frequency and location but where both parameters are measured as functions of solar flux. $N m \mathrm{~F} 2$ increases linearly with solar flux. Occurrence of L-band scintillation S4 also increases linearly with solar flux, and so is consistent with its linear dependence on $N m \mathrm{~F} 2$. The importance here is that the finding is not limited to the technique of measurement used here nor to solar maximum.

The third study, observations from Taipei, finds occurrence of $137 \mathrm{MHz}$ scintillation to increase as a linear function of $N m$ F2. Its importance is yet a third technique that shows dependence consistent with that found at $1.5 \mathrm{GHz}$, but in the very different frequency regime of $137 \mathrm{MHz}$.

The importance of these results is that scintillation is caused by the bubble, but its magnitude is determined by the magnitude of the electron density that the bubble intersects. As a result, because bubbles are unpredictable, if $N m \mathrm{~F} 2$ can be predicted, the maximum possible S4 may be predictable. This work, which is the first attempt at such a prediction of the upper limit to amplitude scintillation, will require a larger data set from different locations, seasons and solar fluxes.

Acknowledgements. The author thanks the following: the team from the Air Force Research Laboratory, Hanscom Research Site, who undertook the expedition to Ascension I.; Keith Groves for the scintillation measurements, and Terrence Bullett and Howard Kunzeler for the ionospheric soundings; Eileen MacKenzie of Boston College Institute for Scientific Research, for the scintillation data and its interpretation.

Topical Editor K. Kauristie thanks E. Correia and E. Miller for their help in evaluating this paper.

\section{References}

Aarons, J.: 50 years of radio-scintillation observations, IEEE Antennas and Propagation Magazine, 39, 7-12, 1997.

Aarons, J., Mullen, J. P., Whitney, H. E., and MacKenzie, E. M., and Basu, S.: Microwave equatorial scintillation during solar maximum, Radio Sci., 16, 939-945, 1981.

Basu, S. and Groves, K. M.: Specification and forecasting of outages on satellite communication and navigation systems, AGU Space Weather Monograph 125, 423-430, 2001.

Basu, S., MacKenzie, E., and Basu, Su.: Ionospheric constraints on VHF/UHF communication links during solar maximum and minimum periods, Radio Sci., 23, 363-378, 1988.

Basu, S., McClure, J. P., Sunanda Basu, Hanson, W. B., and Aarons, J.: Coordinated study of equatorial scintillation and in situ and radar observations of nighttime $\mathrm{F}$ region irregularities, J. Geophys. Rev., 85, 5119-5130, 1980.

Basu, Sunanda, Basu, S., McClure, J. P., Hanson, W. B., and Whitney, H. E.: High resolution in situ data of electron densities and VHF/GHz scintillations in the equatorial region, J. Geophys. Res., 88, 403-415, 1983. 
Briggs, B. H. and Parkin, I. A.: On the variation of radio star and satellite scintillation with zenith angle, J. Atmos. Terr. Phys., 25, 339-365, 1963.

Hysell, D. L. and Kelley, M. C.: Decaying equatorial F region plasma depletions, J. Geophys. Res., 102, 20007-20017, 1997

Huang, C.-M.: F-region irregularities that cause scintillations and spread-F echoes at low latitude, J. Geophys. Res., 75, 48334841, 1970.

Kudeki, E. and Bhattacharyya, S.: Postsunset vortex in equatorial F-region plasma drifts and implication for bottomside spread-F, J. Geophys. Res., 104, 28163-28170, 1999.

Reinisch, B. W., Bibl, K., Kitrosser, D. F., Sales, G. S., Tong, J. S., Zahn, Z.-M., Bullett, T. W., and Rails, J. A.: The digisonde 256 ionospheric sounder, in: World Ionosphere/Thermosphere Study, edited by: Liu, C. H., WITTS Handbook, vol. 2, SCOSTEP, IL, pp. 350-366, 1989.
Whalen, J. A.: An equatorial bubble: Its evolution observed in relation to bottomside spread $\mathrm{F}$ and to the Appleton anomaly, J. Geophys. Res., 105, 5303-5315, 2000.

Whalen, J. A.: Linear dependence of the postsunset equatorial anomaly electron density on solar flux and its relation to the maximum prereversal $E \times B$ drift velocity through its dependence on solar flux, J. Geophys. Res., 109, A07309, doi:10.1029/2004JA0105, 2004.

Weber, E. J., Buchau, J., Eather, R. H., and Mende, S. B.: Northsouth-aligned equatorial airglow depletions, J. Geophys. Res., 83, 712-716, 1978.

Weber, E. J., Brinton, H. C., Buchau, J., and Moore, J. G.: Coordinated airborne and satellite measurements of equatorial plasma depletions, J. Geophys. Res., 87, 10503-10513, 1982. 